\title{
Size-dependent cellular uptake of exosomes
}

Federica Caponnetto ${ }^{a, b}$, Ivana Manini ${ }^{b}$, Miran Skrap ${ }^{c}$, Timea Palmai-Pallaga ${ }^{a}$, Carla Di Loreto ${ }^{b}$, Antonio Paolo Beltrami ${ }^{\mathrm{b}}$, Daniela Cesselli ${ }^{\mathrm{b}}$, Enrico Ferrari ${ }^{\mathrm{a}^{*}}$

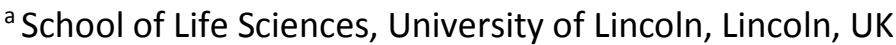

${ }^{\mathrm{b}}$ Department of Medical and Biological Sciences, University of Udine, Udine, Italy.

${ }^{c}$ Neurosurgery Unit, Department of Neuroscience, Santa Maria della Misericordia University Hospital, Udine, Italy.

"Corresponding author: Enrico Ferrari, School of Life Sciences, University of Lincoln, Brayford Pool, LN6 7TS Lincoln, UK. Telephone: +441522886302; E-mail: eferrari@lincoln.ac.uk

\begin{abstract}
The ability of exosomes to elicit specific cellular responses suggests that they may be increasingly used as therapeutics. Their vesicular nature makes them suitable as potential nanocarriers for drugs or nucleic acids delivery. Here we address the question whether the method of preparation of enriched exosomal fractions can affect their uptake by cells and their ability to trigger a response.

We compared ultracentrifugation and polymer-based precipitation methods on supernatants of glioma-associated stem cells isolated from a high-grade glioma patient. We determined particle size distributions after purification and their correlation with uptake, proliferation and migration in glioblastoma cell cultures.
\end{abstract}

Our findings indicate that polymer-based precipitation leads to smaller particle size distributions, faster uptake by target cells and increased cellular motility. The different effect that isolation method-dependent populations of particles have on cell motility suggests their size distribution could also profoundly affect exosomes therapeutic potential.

Keywords: Exosomes; Glioma; GASC; Cellular Uptake; AFM; Flow Cytometry 
Abbreviations: GASC=Glioma-Associated Stem Cells; DiD=1,1'-dioctadecyl-3,3,3',3'-

tetramethylindodicarbocyanine perchlorate; SDS-PAGE=sodium dodecyl sulfate polyacrylamide gel electrophoresis

Funding: Timea Palmai-Pallag was funded by the BBSRC grant BB/K019597/1.

Conflict of interest: no competing interests are present.

Word count: abstract (143), manuscript (5051); References: 38; Tables/Figures: 6 


\section{Background}

Exosomes are extracellular membrane vesicles found in many body fluids and released by a variety of cell types $[1,2]$. It has been shown that exosomes play an active role in cell-to-cell communication [3-6] and, for example, contribute to determine the tumour micro-environment with consequences for proliferation, invasion and metastasis [7]. Much attention has been focused on the molecular characterization of exosomes content, primarily nucleic acids and proteins, and on the specific markers exposed on the lipid vesicles that determine specific interactions with target cells [8]. However, it has been suggested that physical properties of the particles may also affect the way exosomes mediate intercellular communication [9]. In fact, in the case of engineered nanoparticles, it has been shown that their size may affect the uptake efficiency and kinetics, the internalization mechanism and also the subcellular distribution [10]. Whether the size of natural vesicles might be also an essential factor that determines how easily they can diffuse in a tissue and how effectively cells can take them up is still unknown and represents the overarching question behind this study.

Exosomes size is reported to vary from 30 to $100 \mathrm{~nm}[1,2]$ and, as a consequence of the small nature of particles, an accurate estimation tends to be elusive. Several techniques are available to determine the size of nanoparticles that have been applied to characterise exosomes. These include Transmission Electron Microscopy (TEM), Atomic Force Microscopy (AFM), Nanoparticle Tracking Analysis (NTA) and Dynamic Light Scattering (DLS) among the most used [11-17]. Unfortunately, none of these methods on its own has the ability to estimate the size distribution accurately and at the same time provide high-throughput. Microscopy techniques, TEM and AFM, provide a direct measurement of individual particles size but generally on samples with small numbers of particles, whereas light scattering techniques, NTA and DLS, quickly provide size distributions based on much larger sample size, however, the size estimation is not direct and prone to artefacts. There is also a degree of diversity in the methods used for the enrichment of the exosomal fractions, mostly based on precipitation by ultracentrifugation or polymers [13]. Different methods can lead to preparations 
with different properties, including a different size distribution and possibly a proportion of extracellular vesicles other than exosomes $[9,18]$.

A comprehensive and accurate understanding of the actual size distribution of exosomes preparations might not be a priority for functional studies or biomarker discovery, but it is of vital importance when moving towards the use of exosomes as therapeutics [19]. In fact it is known that the physical properties of lipid vesicles, including size, can have a dramatic effect on cells ability to internalise them [20]. Therefore, a better knowledge of the effect of extraction method on particles size distribution and cellular uptake would be beneficial in this context and could also contribute to the general understanding of the mechanism of exosomes uptake in intercellular communication [21]. Apart from their potential use as therapeutics, exosomes could be used as predictors of disease status [22]. For example, Glioma-Associated Stem Cells (GASC), a population of stem cells isolated from the glioma microenvironment and endowed with a significant prognostic potential, exert their tumour supporting activity through the release of exosomes [23]. Also in this context, besides the already well-studied molecular profiles, a better understanding of the physical properties of exosomes might be informative and useful to predict the clinical outcome of diseases [24].

Here we purified exosomal fractions from GASC supernatants, derived from a high-grade glioma patient stem cells, using two well established and widely used methods: ultracentrifugation (UC) and ExoQuick (EQ) precipitation $[9,13,18,25]$. We then accurately characterised the resulting particles size distribution using primarily AFM and NTA. We exposed A172 glioblastoma cell cultures to set amounts of fluorescently labelled exosomes and evaluated the cellular uptake by fluorescence microscopy and flow cytometry. We finally verified whether differential uptake of exosomes preparations could affect glioma cell motility. 


\section{Methods}

\section{Cell cultures}

Human glioma samples were collected at the Department of Neuroscience, Santa Maria della Misericordia University Hospital in Udine, after a written informed consent from the patient was obtained, in accordance with the declaration of Helsinki, and with approval by the independent ethics committee of the hospital. Glioma associated stem cells (GASC) and A172 human glioblastoma cells were cultured as described in $[26,27]$ and detailed in the Supplementary methods.

\section{Exosomes purification, size and density measurements}

A172 and GASC supernatants were processed using either ExoQuick (EQ) according to manufacturer's protocol or ultracentrifugation (UC) precipitation as previously described [13]. Size and density measurements were performed using AFM, NTA and DLS. Purification, characterization methods and protein assays for density estimation are detailed in the Supplementary methods section.

\section{Exosomes uptake assays}

Purified exosomes were labelled by DiD (Life Technology) and re-precipitated to remove the excess of fluorophore. Uptake was analyzed by confocal or epi-fluorescence microscopy as detailed in Supplementary methods. Flow cytometry was performed on a FACSverse flow cytometer (BD Biosciences) on cells detached from substrates with TrypLE Express solution (Life Technologies), washed and resuspended in $200 \mu$ I PBS. After reaching a count of $1 \times 10^{4}$ cells, the data were gated on the basis of forward and side scattering and the mean fluorescence intensity (MFI) was calculated as the ratio between total DiD fluorescence over the number of cells showing fluorescence for both DiD and Hoechst. 


\section{Scratch assay}

On a 24 -well plate $1 \times 10^{5}$ cells were seeded and brought to high cellular confluence then scratched using a $200 \mu \mathrm{l}$ tip. Phase contrast images of the scratches were acquired every 6 hours, until their complete closure, by a Nikon Eclipse TS100 microscope (Nikon) and a 10x objective. The velocity ( $\mu \mathrm{m} /$ hours) was calculated by measuring the distance covered into the scratch by the front of migration in several points. Images were processed and distances calculated using ImageJ (National Institutes of Health).

Statistical analysis of size, uptake and migration measurements is detailed in the Supplementary methods.

\section{Results}

\section{Effect of the extraction method on the size distribution of exosomal fractions}

EQ and UC isolated exosomes from three GASC cultures supernatants derived from the same patient were adsorbed on mica coverslips and imaged using AFM to estimate the size distribution of the particles in the exosomes fractions. Figure $1 A$ and $B$ are representative $5 \times 5 \mu m$ images of $E Q$ and UC purified exosomes respectively, obtained from the same supernatant. Although both preparations produced individually separated particles with a near-spherical morphology, the EQ method generated particles that appeared to be smaller. This was confirmed by a quantitative analysis on a large number of particles from 10 images acquired from the two preparations. Supplementary figure $1 \mathrm{~A}$ shows the size distributions in which the size of individual particles was determined from the maximum height of each particle. The analysis of the distributions confirmed that EQ purification leads to smaller particles than UC and the resulting peak size average over the three replicates, reported in Table 1, confirmed the significant difference in particle size of extracellular vesicles obtained with the two methodologies. To investigate whether this observation was limited to the case of GASC only or could be a more general effect due to the nature of the extraction method, we repeated the experiment using the same exact approach on exosomes extracted from three 
supernatants of A172 glioblastoma cell cultures. Also in this case the results confirmed that exosomes isolated by EQ were significantly smaller than those obtained by UC $(p<0.05$, Figure $1 C$ and D, Table 1 and Supplementary figure 1B).

Interestingly, we found that the size distribution, measured by the widely used NTA technique on the same preparations, failed to show any substantial difference between the two extraction methods both in GASC and A172 preparations (Table 1 and Supplementary figure 2). This is likely due to the fact that the smallest particles in EQ preparations are too small to provide sufficiently intense scattering for the detection by the NTA instrument camera. In fact it has been previously observed that the limit of detection of nanovesicles using NTA is about $50 \mathrm{~nm}$ [28], which is close to the size of EQ exosomes measured by AFM.

To verify this hypothesis, we first measured the density of EQ and UC particles adsorbed on mica (particles $/ \mu \mathrm{m}^{2}$ ) from the entire datasets of Supplementary figure 1 and we compared these values with the particle density in solution measured by NTA (particles $/ \mathrm{ml}$ ). We found that the ratio calculated from AFM density over NTA density was larger in EQ preparations, both for GASC and A172 supernatants (Supplementary table 1). This suggests that NTA underestimates the number of particles per volume unit when their size is below a certain threshold, which is more frequent in EQ preparations. This is supported by a further computation made on the AFM dataset of Supplementary figure 1 , on which we re-calculated the particle density, this time limited to particles larger than $50 \mathrm{~nm}$, and found that the proportion AFM density/NTA density became very close for EQ and UC preparations, both for GASC and A172 samples (Supplementary table 1), suggesting that the particles smaller than $50 \mathrm{~nm}$ are likely responsible for the mismatch between AFM and NTA measured densities.

We attempted size estimation of GASC EQ and UC exosomes by DLS and found peaks at $41.5 \pm 3.2$ $\mathrm{nm}$ and $90.1 \pm 0.7 \mathrm{~nm}$ respectively. Although these values were consistent with AFM measurements, we also reported major intensity contribution from apparent peaks at about $500 \mathrm{~nm}$ (Supplementary 
figure 3). These are likely due to the inevitable presence of debris from preparations and lead to large polydispersity indexes of about 0.7 , which suggest that the samples are not suitable for accurate DLS measurements.

To verify whether the apparent cut-off in the size distributions of UC extracted exosomes can be attributed to the inability of UC to sediment the smallest particles, we re-precipitated an A172 EQ preparation having a peak size of $45.3 \mathrm{~nm}$ using the UC method and found that the size distribution peak measured from a new set of AFM images shifted to $85.0 \mathrm{~nm}$, which is consistent with values obtained for samples directly extracted with UC (Table 1 ). In addition, the particle density measured on AFM datasets decreased, from 3.8 particles $/ \mu \mathrm{m}^{2}$ of the original EQ preparation to 1.1 particles $/ \mu \mathrm{m}^{2}$ after re-precipitation with UC, suggesting that the smallest particles were lost in the process.

Together, these results suggest that the method of enrichment leads to different populations of particles being extracted and EQ method precipitates an exosomal fraction, which is richer in smaller particles than those obtained with UC. Also, for this reason, NTA is not suitable for the accurate estimation of the size distribution of exosomes extracted with EQ.

\section{Estimation of particle density in exosomes preparations}

Morphological examination of AFM images, such as those in Figure 1, and density measurement with both AFM and NTA (Table 1) suggest that EQ precipitation leads to larger densities of particles compared to UC. We were not able to make use of methods based on protein determination to estimate the particle density of each preparation, as EQ preparations tend to precipitate serum proteins from the culture media along with the exosomes, resulting in a high background that makes Bradford and BCA colorimetric assays or SDS-PAGE impractical (Table 1 and Supplementary figure 4). We then used fluorescence intensity from an anti CD9 ELISA assay in combination with NTA data to determine the best estimate of exosomes density in all the preparations. We found that EQ preparations result in less intense fluorescence signal associated to CD9, especially in the case of 
GASC supernatants. This is in contrast with the previous observation that EQ leads to larger amounts of particles compared to UC, suggesting that not all the particles precipitated by EQ are of exosomal nature or present equal levels of CD9.

Although NTA failed to give an accurate estimate of the size distribution in the case of EQ extracted exosomes, based on the CD9 data, we considered the density estimation by NTA sufficiently accurate to be used when normalising the number of particles to use in subsequent uptake experiments. Also, from the NTA particle density data of the sample analysed in Supplementary table 1, it is clear that NTA was able to detect the higher concentration of particles in the EQ preparation, well visible by AFM. Nevertheless, when drawing conclusions on the results reported below, we took into account the risk of underestimating the number of particles in EQ preparations based on the inability of NTA to detect the smallest particles, which is mitigated by the CD9 data (see discussion).

\section{Cellular uptake of exosomal fractions with different size distributions}

To assess whether different size distributions observed for EQ and UC exosomes can affect cellular uptake, we labelled particles of both preparations with the lipophilic dye DiD and exposed A172 cell cultures to the labelled exosomes for 1, 3, and 6 hours. Confocal images on live cells confirmed that exosomes internalization is well visible at this concentration $\left(0.5 \times 10^{9}\right.$ exosomes, as estimated from NTA measurement, in approximately 20,000 cells/petri dish). However, we observed that, whereas EQ exosomes uptake was already very extensive within 3 hours, UC preparations needed 6 hours to show a comparable uptake. Figure 2 shows representative images of the uptake assay at different times. The images were acquired using the same setup and the same imaging parameters, therefore we concluded the higher intensity localised in intracellular compartments observed in A172 cultures exposed to GASC EQ exosomes is most likely due to a more efficient uptake of particles that are smaller than in the UC samples. 
To extend the observation to exosomes having an origin other than GASC, we repeated the experiment on A172 cells using exosomes extracted from A172 cells themselves and observed the same preferential uptake of EQ preparations (Supplementary figure 5).

To support the qualitative observations above, we used FACS to estimate the mean fluorescence intensity on large numbers of A172 cells. We limited the calculation to the cells showing fluorescence intensity of Hoechst nuclear staining above a threshold likely determining the subpopulation of living cells (Figure 3). The results confirm the preferential uptake of GASC EQ exosomes compared to UC preparations. This was also the case for A172 extracted exosomes (Supplementary figure 6).

To verify whether uptake saturates at similar levels for EQ and UC exosomes over time, A172 cells were exposed to DiD-labelled GASC exosomes for a time interval ranging from 1 to 72 hours (Figure 4). Although at 72 hours the fraction of DiD-positive cells was the same for EQ and UC exosomes, at 6, 24 and 48 hours EQ uptake was more extensive, indicating different uptake kinetic characteristics of the two preparations.

To exclude that DiD labelling of target cells was due to the simple transfer of the lipophilic dye from the membrane of exosomes to the cell membrane, exosomes were loaded with a fluorescent oligonucleotide. Supplementary figure 7 shows the cytoplasmic localisation of the labelled oligonucleotide after exposure to exosomes, confirming that exosomes were able to transfer their cargo to A172 target cells.

Additionally, to rule out the possibility that the higher fluorescence intensity observed in EQ uptake was due to more extensive incorporation of DiD in EQ exosomes compared to UC, we quantified the fluorescence intensity of known densities of exosomes extracted with the two methods. We found that DiD staining of EQ preparations resulted into slightly less intense fluorescence (Supplementary table 2). This suggests that higher intensities in uptake experiments involving EQ exosmes are most 
likely due to larger number of internalised particles, rather than higher average fluorescence intensity of particles.

To verify whether the preferential uptake of $\mathrm{EQ}$ exosomes was determined by the different size distribution, rather than the nature of the precipitation itself, we manipulated the size distribution by re-precipitation of A172 EQ exosomes using UC, followed by DiD labelling. As expected, the peak size of the distribution measured by AFM increased from approximately $50 \mathrm{~nm}$ to nearly $100 \mathrm{~nm}$, in line with the data of Table 1 . The cellular uptake after 6 hours was significantly reduced in comparison to a control EQ extract that was not re-precipitated by UC (Supplementary figure 8). These results suggest that EQ extracted exosomes, once size-selected using an extra UC precipitation step, are larger in size and less effectively internalised by A172 cells. We also performed another similar double-precipitation extraction, but we first extracted A172 exosomes by UC and then reprecipitated them by EQ, followed by DiD labelling. Confocal images of the uptake of EQ reprecipitated UC exosomes, compared to regular UC preparation, show that the EQ extra precipitation step does not lead to any increase in the uptake (Supplementary figure 9). We also quantitatively confirmed the results by flow cytometry (Supplementary figure 10).

Moreover, to exclude that the increased uptake of EQ exosomes was related to the serum proteins precipitated together with the exosomes that are visible in Supplementary figure 3, UC exosomes were prepared as usual, but resuspended using Exoquick-precipitated culture medium (Supplementary figure 11). The presence of Exoquick-precipitated serum proteins did not increase the UC exosomes uptake, excluding that this could be due to contaminants from EQ preparations. All these results together suggest that the kinetic of uptake of exosomes by A172 cells is significantly influenced by the enrichment method and seems to be strictly associated with the average size of particles. 


\section{Functional assays on exosomes-exposed cell cultures}

A172 cell cultures are known to show higher motility rates upon exposure to GASC extracted exosomes [23]. We wanted to verify whether the preferential uptake of GASC exosomal fractions extracted with EQ was able to trigger a more prominent response compared to UC extractions. We conditioned equal amounts of cells for 48 hours with $0.5 \times 10^{9}$ GASC extracted EQ and UC exosomes (density measured by NTA). We then opened a gap in the otherwise confluent cell cultures by scratching the petri dish with a tip and measured the velocity $(\mu \mathrm{m} / \mathrm{h})$ at which the cell cultures were able to fill the gap. The results reported in Figure 5 clearly show that the cell cultures conditioned with EQ exosomes were significantly quicker at filling the gap, compared to the cultures exposed to UC exosomes or the control (no exposure to exosomes). To establish whether the functional effect could be related to the density of exosomes at which A172 cells were exposed, we repeated the assay at densities ranging from tenfold excess of the concentration of Figure $5 \mathrm{M}$, down to a 10,000 times dilution of the same. As shown in Figure $5 \mathrm{~N}$, a highly significant linear trend between exosomes dose and motility was observed.

This suggests that the preferential uptake of EQ preparations also translates into a higher motility and the intensity of the response to the "message" delivered is most likely proportional to the number of particles that were internalised during the conditioning phase.

\section{Discussion}

In this work, we emphasised the importance of looking at the physical properties of exosomal preparations rather than limiting the characterisation to molecular attributes. Our rationale is that exosomes play an important role in cellular communication and, regardless of the specific molecules they carry, the nature of the "envelope" might be of great importance for the delivery of the message. For example, in the case of nanoengineered nanoparticles it has been shown that size per se can affect their interaction with live cells and $40-50 \mathrm{~nm}$ was identified as the diameter range characterised by the highest cell uptake level [10]. As this has never been investigated for exosomes, 
we focused our study on exosomes size, as this is the most evident physical property that can have an effect on the way the vesicles diffuse in the tissues and are taken up by cells. Alas, to investigate the mechanism by which these exosomes are internalised was not in the scope of the present study.

To carefully estimate the size distribution of GASC exosomes, we compared two widely used methods, AFM and NTA, and found that although the latter can be used to estimate particles density, it is not sufficiently accurate to determine the exact size distribution of all the exosomal fractions of interest, as it failed to detect the smallest particles especially in EQ samples. We confirmed previous observations that the technique is not suitable for lipid particles smaller than 50 $\mathrm{nm}$ [28], which instead are accessible to AFM. Similarly, DLS failed to provide useful size information, as the technique is generally not suitable when the samples present even a small amount of debris, which scatter more intensely than the majority of the other particles in solution. However, due to the high sensitivity of the detector, compared to the NTA camera, the DLS instrument was able to detect a peak that likely correspond to the exosomes. Moreover, both NTA and DLS measure the hydrodynamic diameter from the scattering of particles in solution, which are very sensitive to the accurate estimation of the temperature and the viscosity of the fluid. AFM instead measure the "dry state" size of the particles and is less affected by the boundary conditions.

The size estimation based on the AFM height profile of particles might be prone to a slight underestimation of the exact diameter of individual exosomes, as it is known lipid vesicles can undergo deformation when adsorbed on mica surfaces and acquire a flattened rather than spherical shape [29]. However, analysis of our AFM datasets shows that the particle diameter calculated from the height is not significantly different from the diameter computed from the volume of the same particle, assuming spherical shape (data not shown). This means that the deformation was minimal and the estimation was accurate. Despite these measurements support the idea that exosomes are quasi-spherical, AFM images show that the particles present sometimes slightly irregular shapes. 
However, the resolution of the images was limited by the size of the tip, which was around $4 \mathrm{~nm}$, therefore it was not possible to systematically study exosomes shape.

It is important to point out that, regardless of the method used to estimate particle size distributions, it is safer to use EQ and UC sizes as a way to compare between extraction methods, rather than provide an absolute estimation of their true size. In fact, the distributions inevitably reflect the conditions in which they were measured, like for example the presence of serum proteins in the media that can form a "corona" surrounding the particle and potentially contribute to an increase of the hydrodynamic diameter of about $10 \mathrm{~nm}[30]$.

Due to the detection limit mentioned in the results, NTA is not able to provide a precise estimate of particles density either. However, our data on CD9 marker suggest that NTA did not underestimate exosome content in EQ samples as the CD9 signal was not larger than UC. It has been shown before that tetraspannin are not equally expressed by different exosomes subpopulations [31] which might also be the reason of our observation.

We also found that NTA is far more accurate at determining exosomes density than protein-based methods. In fact, especially in exosomes samples prepared by chemical precipitation, the presence of large amounts of proteins from the serum makes exosomal proteins estimation impractical. The use of common exosomal markers like CD9, CD63 and CD81 is an appropriate alternative for NTA and would minimise the risk to overestimate the number of exosomes in a preparation by including non-exosomal vesicles. However this approach would be applicable only to qualitatively compare the density of exosomes purified from the same source, as it would not be reasonable to assume that exosomes from different sources carry the same amount of each marker [32,33].

Importantly, we found that two of the common methods of exosomes purification lead to significantly different particle size distributions when assessed by AFM. Specifically, EQ purification generates particles that are on average smaller than UC exosomes. Although this might be irrelevant when characterising molecular markers associated with exosomes, it becomes very important in the 
case of experiments involving cellular uptake [34]. Our results show that the uptake by A172 cells of GASC exosomes isolated with different methods follows a different kinetics, with EQ exosomes showing faster uptake than UC. We demonstrated that this is associated with the size distribution by using double precipitations as controls and we excluded a possible effect of contaminants, such as serum proteins, in EQ exosomes preparations.

In recent literature, the impact of isolation methods on purification yield, physical properties and biological content has been investigated in several exosomes preparations. UC exosomes from saliva were found to be smaller than EQ as well as different in morphology and protein content [18]. UC exosomes isolated from cancer cell supernatants were also found larger than those purified by density gradient from the same source [35]. Density gradient was evaluated as the best method to study exosomes RNA, although the method is characterized by lower yields when compared to UC and EQ [9]. Recent literature highlights differences between purification methods, without focussing on the effect on uptake, with the exception of a study assessing concentration and time dependence of UC exosomes uptake by bladder cancer cells [36]. To our knowledge, our work is the first study comparing cell uptake of two different preparations and investigating the functional effects of the differential uptake in term of target cell motility.

Our findings demonstrated that the isolation method has an influence on physical parameters such as size distribution and, ultimately, different size distributions of GASC exosomes lead to significantly different effects on the motility of glioblastoma cells. Opposite to the purely size-dependent uptake of the engineered nanoparticles mentioned above [10], we cannot exclude that exosomes uptake and functional effects from our two exosomes populations can be also affected by differences in properties other than size, both physical (e.g., electrochemical membrane potential) and biological (e.g., surface proteins, molecular cargos). Nevertheless, our data suggest that size can determine different uptake kinetics and biological functions. 
Based on this study, the recommended method of purification, in case subsequent studies require cellular uptake, would be EQ. In fact, smaller EQ exosomes size leads to faster uptake compared to UC and eventually triggers a more prominent functional effect on cell cultures (motility in this case).

With regards to uptake, it will be key to investigate further the specific mechanism of entry of exosomes, to establish whether size and/or method of extraction could make any difference. In fact, exosomes can either fuse to the cell membrane or deliver their biological content through the endocytic pathway, including both clathrin-dependent and independent endocytosis [37]. Preliminary data in our laboratory suggest that exosomes gain entry into target cells via more than one route (data not shown), making necessary to design future experiments to investigate the specific contribution of each mechanism of entry.

Apart from the practical considerations above, the observation that cells preferentially uptake smaller exosomes which also trigger a larger response has a potential general impact in therapies involving exosomes [38]. For example, according to our findings, the therapeutic use of exosomes could be enhanced by using size as a parameter to select functionally different sub-populations. This study also opens further questions whether different cell types produce exosomes of different average size and whether this may affect the way they contribute to the shaping of their microenvironment. The results in this work suggest the hypothesis that cell types producing smaller exosomes might be more effective at delivering their message. It has been shown that GASC exosomes isolated from glioma patients are heavily involved in supporting the tumour progress and that this effect is proportional to the aggressiveness of the disease. However, it is not known whether their exosomes size plays a role in this process. While a few research reports differences in exosomes size and size distribution between normal and malignant cells, a study to address the question of whether there is a correlation between the size distribution of exosomes and their influence on the ability to trigger alternative cellular responses specific to cell type or 
pathophysiological condition and statuses is missing. Such comparative systematic study would significantly benefit from the findings described here. 


\section{References}

[1] Mathivanan S, Ji H, Simpson RJ. Exosomes: Extracellular organelles important in intercellular communication. J Proteomics 2010;73:1907-20.

[2] Vlassov A V, Magdaleno S, Setterquist R, Conrad R. Exosomes: Current knowledge of their composition, biological functions, and diagnostic and therapeutic potentials. Biochim Biophys Acta - Gen Subj 2012;1820:940-8.

[3] Valadi H, Ekström K, Bossios A, Sjöstrand M, Lee JJ, Lötvall JO. Exosome-mediated transfer of mRNAs and microRNAs is a novel mechanism of genetic exchange between cells. Nat Cell Biol 2007;9:654-9.

[4] Mathivanan S, Fahner CJ, Reid GE, Simpson RJ. ExoCarta 2012: database of exosomal proteins, RNA and lipids. Nucleic Acids Res 2012;40:D1241--4.

[5] Schageman J, Zeringer E, Li M, Barta T, Lea K, Gu J, et al. The complete exosome workflow solution: from isolation to characterization of RNA cargo. Biomed Res Int 2013;2013:253957.

[6] Simons M, Raposo G. Exosomes--vesicular carriers for intercellular communication. Curr Opin Cell Biol 2009;21:575-81.

[7] Suntres ZE, Smith MG, Momen-heravi F, Hu J, Zhang X, Wu Y, et al. Therapeutic Uses of Exosomes. Exosomes Microvescicles 2013;1:1-8.

[8] Raposo G, Stoorvogel W. Extracellular vesicles: Exosomes, microvesicles, and friends. J Cell Biol 2013;200:373-83.

[9] Van Deun J, Mestdagh P, Sormunen R, Cocquyt V, Vermaelen K, Vandesompele J, et al. The impact of disparate isolation methods for extracellular vesicles on downstream RNA profiling. J Extracell Vesicles 2014;3:1-14.

[10] Shang L, Nienhaus K, Nienhaus G, Astruc D, Saha K, Agasti S, et al. Engineered nanoparticles 
interacting with cells: size matters. J Nanobiotechnology 2014;12:5.

[11] Kesimer M, Gupta R. Physical characterization and profiling of airway epithelial derived exosomes using light scattering. Methods 2015.

[12] van der Pol E, Hoekstra a. G, Sturk A, Otto C, Van Leeuwen TG, Nieuwland R. Optical and nonoptical methods for detection and characterization of microparticles and exosomes. J Thromb Haemost 2010;8:2596-607.

[13] Théry C, Amigorena S, Raposo G, Clayton A. Isolation and characterization of exosomes from cell culture supernatants and biological fluids. Curr Protoc Cell Biol 2006;30:3.22.1-3.22.29.

[14] Hoen ENMNT, van der Vlist EJ, Aalberts M, Mertens HCH, Bosch BJ, Bartelink W, et al. Quantitative and qualitative flow cytometric analysis of nanosized cell-derived membrane vesicles. Nanomedicine Nanotechnology, Biol Med 2012;8:712-20.

[15] Sharma S, Das K, Woo J, Gimzewski JK. Nanofilaments on glioblastoma exosomes revealed by peak force microscopy. J R Soc Interface 2014;11:20131150.

[16] Witwer KW, Buzás El, Bemis LT, Bora A, Lässer C, Lötvall J, et al. Standardization of sample collection, isolation and analysis methods in extracellular vesicle research. J Extracell Vesicles 2013;2:1-25.

[17] Sokolova V, Ludwig AK, Hornung S, Rotan O, Horn PA, Epple M, et al. Characterisation of exosomes derived from human cells by nanoparticle tracking analysis and scanning electron microscopy. Colloids Surfaces B Biointerfaces 2011;87:146-50.

[18] Zlotogorski-Hurvitz A, Dayan D, Chaushu G, Korvala J, Salo T, Sormunen R, et al. Human salivaderived exosomes: comparing methods of isolation. J Histochem Cytochem 2015;63:181-9.

[19] Gutiérrez-Vázquez C, Villarroya-Beltri C, Mittelbrunn M, Sánchez-Madrid F. Transfer of extracellular vesicles during immune cell-cell interactions. Immunol Rev 2013;251:125-42. 
[20] Sun D, Zhuang X, Zhang S, Deng Z-B, Grizzle W, Miller D, et al. Exosomes are endogenous nanoparticles that can deliver biological information between cells. Adv Drug Deliv Rev 2013;65:342-7.

[21] Christianson HC, Svensson KJ, van Kuppevelt TH, Li J-P, Belting M. Cancer cell exosomes depend on cell-surface heparan sulfate proteoglycans for their internalization and functional activity. Proc Natl Acad Sci U S A 2013;110:17380-5.

[22] Ko J, Carpenter E, Issadore D, Etzioni R, Urban N, Ramsey S, et al. Detection and isolation of circulating exosomes and microvesicles for cancer monitoring and diagnostics using micro/nano-based devices. Analyst 2016;141:450-60.

[23] Bourkoula E, Mangoni D, lus T, Pucer A, Isola M, Musiello D, et al. Glioma-Associated Stem Cells: A Novel Class of Tumor-Supporting Cells Able to Predict Prognosis of Human Low-Grade Gliomas. Stem Cells 2014;32:1239-53.

[24] Borgiani B, Colombo E, Verderio C, Furlan R. Microvesicles: novel biomarkers for neurological disorders. Front Physiol 2012;3.

[25] Lamparski HG, Metha-Damani A, Yao JY, Patel S, Hsu DH, Ruegg C, et al. Production and characterization of clinical grade exosomes derived from dendritic cells. J Immunol Methods 2002;270:211-26.

[26] Beltrami AP, Cesselli D, Bergamin N, Marcon P, Rigo S, Puppato E, et al. Multipotent cells can be generated in vitro from several adult human organs (heart, liver, and bone marrow). Blood 2007;110:3438-46.

[27] Cesselli D, Beltrami AP, D'Aurizio F, Marcon P, Bergamin N, Toffoletto B, et al. Effects of age and heart failure on human cardiac stem cell function. Am J Pathol 2011;179:349-66.

[28] van der Pol E, Coumans F a W, Grootemaat a. E, Gardiner C, Sargent IL, Harrison P, et al. Particle size distribution of exosomes and microvesicles determined by transmission electron 
microscopy, flow cytometry, nanoparticle tracking analysis, and resistive pulse sensing. J Thromb Haemost 2014;12:1182-92.

[29] Egawa H, Furusawa K. Liposome Adhesion on Mica Surface Studied by Atomic Force Microscopy. Langmuir 1999;15:1660-6.

[30] Dominguez-Medina S, McDonough S, Swanglap P, Landes CF, Link S. In Situ Measurement of Bovine Serum Albumin Interaction with Gold Nanospheres. Langmuir 2012;28:9131-9.

[31] Kowal J, Arras G, Colombo M, Jouve M, Morath JP, Primdal-Bengtson B, et al. Proteomic comparison defines novel markers to characterize heterogeneous populations of extracellular vesicle subtypes. Proc Natl Acad Sci 2016;113:E968-77.

[32] Sharma S, Gillespie BM, Palanisamy V, Gimzewski JK. Quantitative Nanostructural and SingleMolecule Force Spectroscopy Biomolecular Analysis of Human-Saliva-Derived Exosomes. Langmuir 2011;27:14394-400.

[33] Zlotogorski-Hurvitz A, Dayan D, Chaushu G, Salo T, Vered M. Morphological and molecular features of oral fluid-derived exosomes: oral cancer patients versus healthy individuals. J Cancer Res Clin Oncol 2015;142:101-10.

[34] Tian T, Wang Y, Wang H, Zhu Z, Xiao Z. Visualizing of the cellular uptake and intracellular trafficking of exosomes by live-cell microscopy. J Cell Biochem 2010;111:488-96.

[35] Zhang Z, Wang C, Li T, Liu Z, Li L. Comparison of ultracentrifugation and density gradient separation methods for isolating Tca8113 human tongue cancer cell line- derived exosomes. Oncol Lett 2014;8:1701-6.

[36] Franzen CA, Simms PE, Van Huis AF, Foreman KE, Kuo PC, Gupta GN, et al. Characterization of uptake and internalization of exosomes by bladder cancer cells. Biomed Res Int 2014;2014:619829. 
[37] Ann Mulcahy L, Charles Pink R, Raul Francisco Carter D. Routes and mechanisms of extracellular vesicle uptake. J Extracell Vesicles 2014;3:24641.

[38] György B, Szabó TG, Pásztói M, Pál Z, Misják P, Aradi B, et al. Membrane vesicles, current state-of-the-art: Emerging role of extracellular vesicles. Cell Mol Life Sci 2011;68:2667-88. 


\section{Table captions}

Table 1. EQ exosomes size distributions are smaller than UC and have higher exosomes densities. Sizes, protein amount and fluorescence intensity are expressed as average \pm standard deviation of three separate preparations. $p$ values are indicated for values that are significantly different.

\section{Figure captions}

Figure 1. EQ exosomes are smaller than UC. (A) and (B) show representative AFM images of GASC EQ and UC exosomes respectively. (C) and (D) show representative images from A172 exosomes extracted using EQ and UC respectively. Scalebars are $1 \mu \mathrm{m}$.

Figure 2. A172 cells show preferential uptake of DiD labelled GASC EQ exosomes. (A-C) Confocal images of cultures exposed to EQ exosomes for up to 6 hours. (D-F) images acquired after the same incubation times using UC exosomes. Fluorescent signals are merged with transmission images (red=DiD emission, blue=Hoechst nuclear stain). Scalebar is $10 \mu \mathrm{m}$.

Figure 3. Flow cytometry data confirm the preferential uptake of GASC EQ exosomes. On the left, representative side scatter (SSC) versus Hoechst dot plot was used to discriminate A172 cells, exposed or not to GASC-derived exosomes, from debris. On the right, histogram overlays showing non-conditioned A172 cells (red) versus A172 cells exposed to DiD-labeled exosomes for 1, 3 and 6 hours (green). The fraction of positive cells and the relative mean fluorescence intensity ratio (MFI) are indicated as insets in each plot. To define cells positive for DiD, a gate including the $1 \%$ most auto-fluorescent population of the non-conditioned cells was drawn. Indicated percentages correspond to the fraction of exosomes-exposed cells within the gate.

Figure 4. EQ exosomes uptake kinetic is faster than UC. (A) Panels show representative pictures of A172 cells incubated for up to 72 hours with DiD-labeled (red) EQ and UC GASC exosomes. Nuclei are stained with DAPI (blue). Scalebar $=50 \mu \mathrm{m}$. (B) Quantitative analysis of the fraction (\%) of A172 cells 
which show uptake of DiD exosomes extracted by EQ (red) and UC (blue), respectively. Data are presented as mean \pm standard deviation and $p<0.05$ is calculated for EQ values versus UC.

Figure 5. The motility of GASC exosomes-exposed A172 cultures is enhanced with EQ preparations. (A-D) Representative images at different times of scratched A172 cell cultures exposed to GASC EQ exosomes. (E-H) Same as A-D but for UC preparations. (I-L) Negative control (NC) consisting of scratched cell cultures not exposed to exosomes. The proliferation is not quick enough to fill the gap within 24 hours. (M) Mean velocity of the front of migration into the scratch. The velocity is calculated over a period of 12 hours and error bars represent the standard deviation of 4 replicates, * $p<0.05$ versus EQ (N) The bar charts values were obtained like in $(\mathrm{M})$ but with a range of exosomes densities ranging from 10X larger down to 10,000 smaller. Error bars represent the standard deviation of 3 replicates.

Figure 1

A

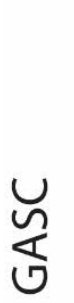

EQ

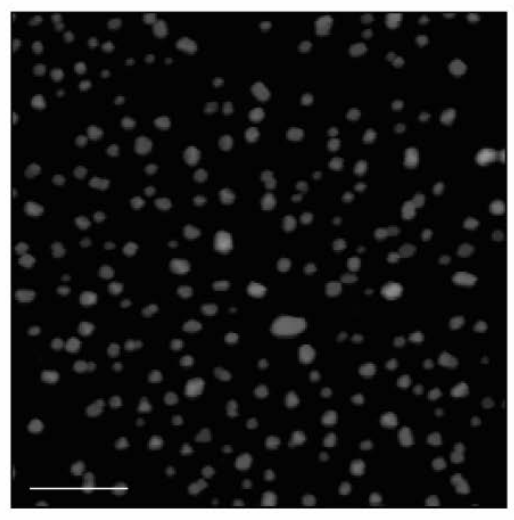

C
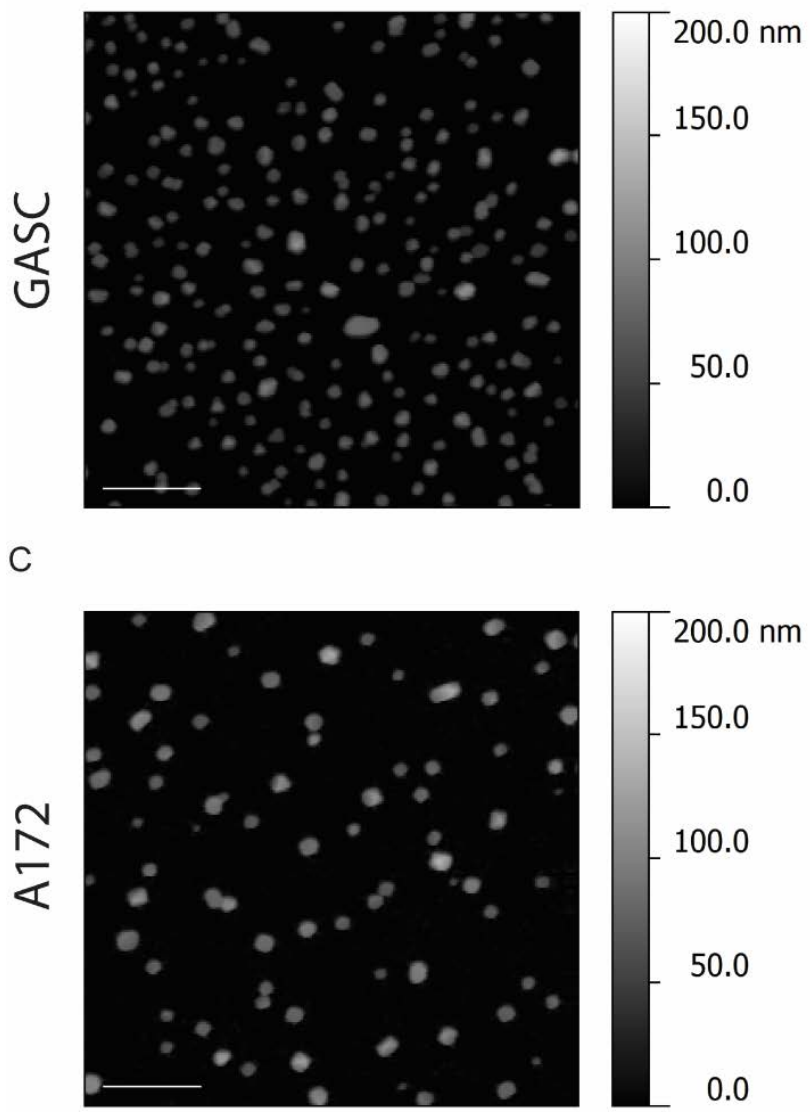

B
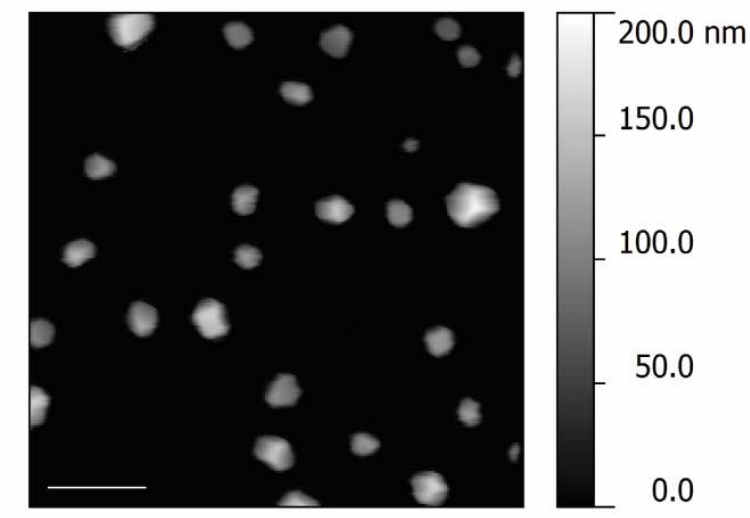

D

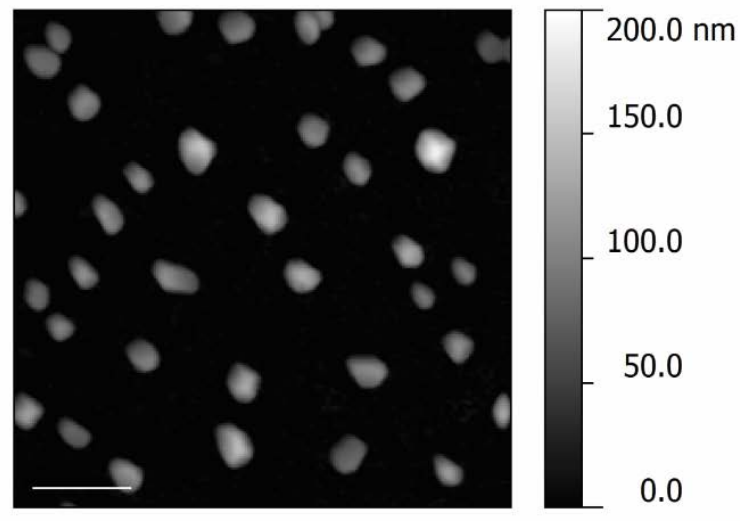


Figure 2

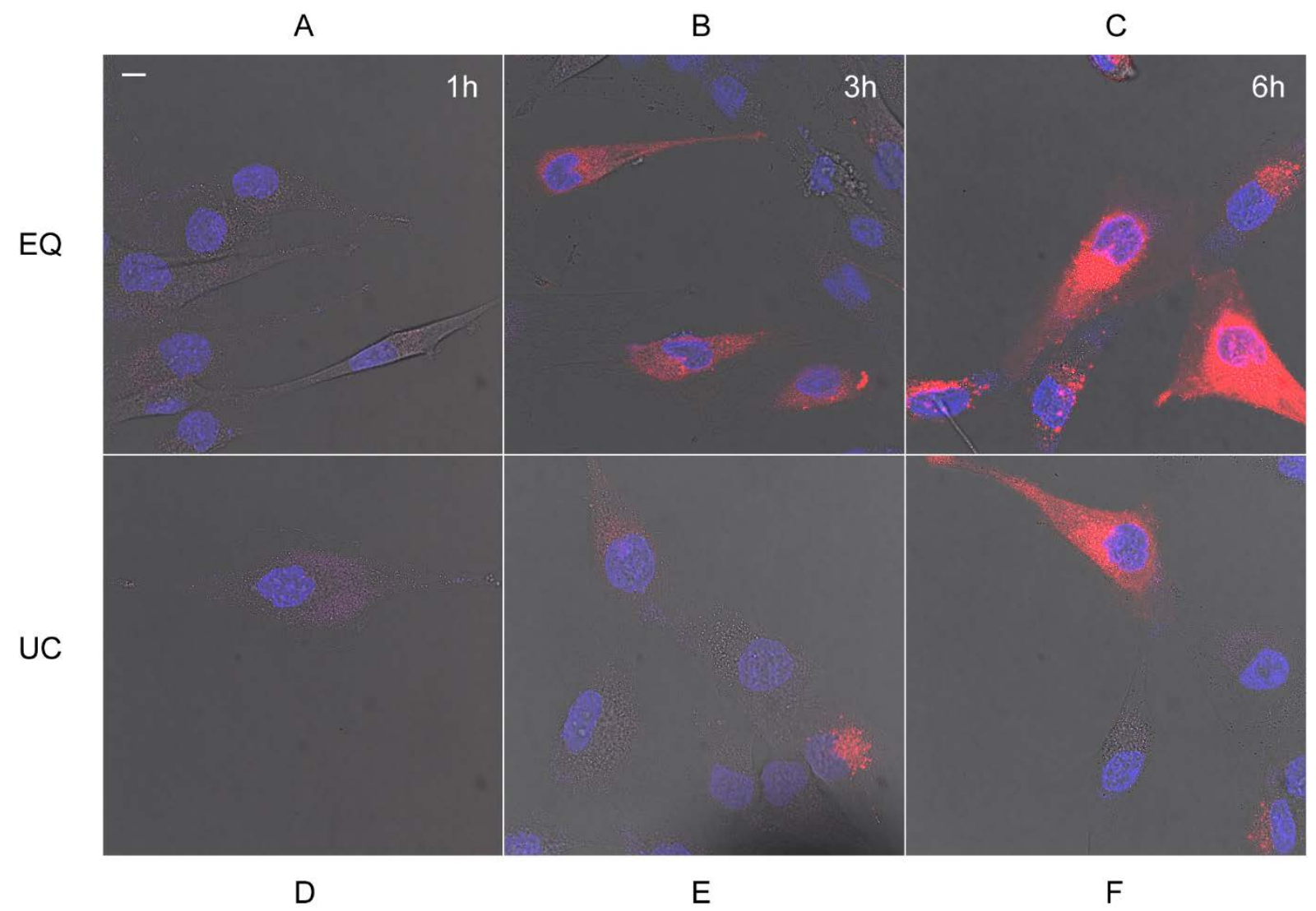

Figure 3

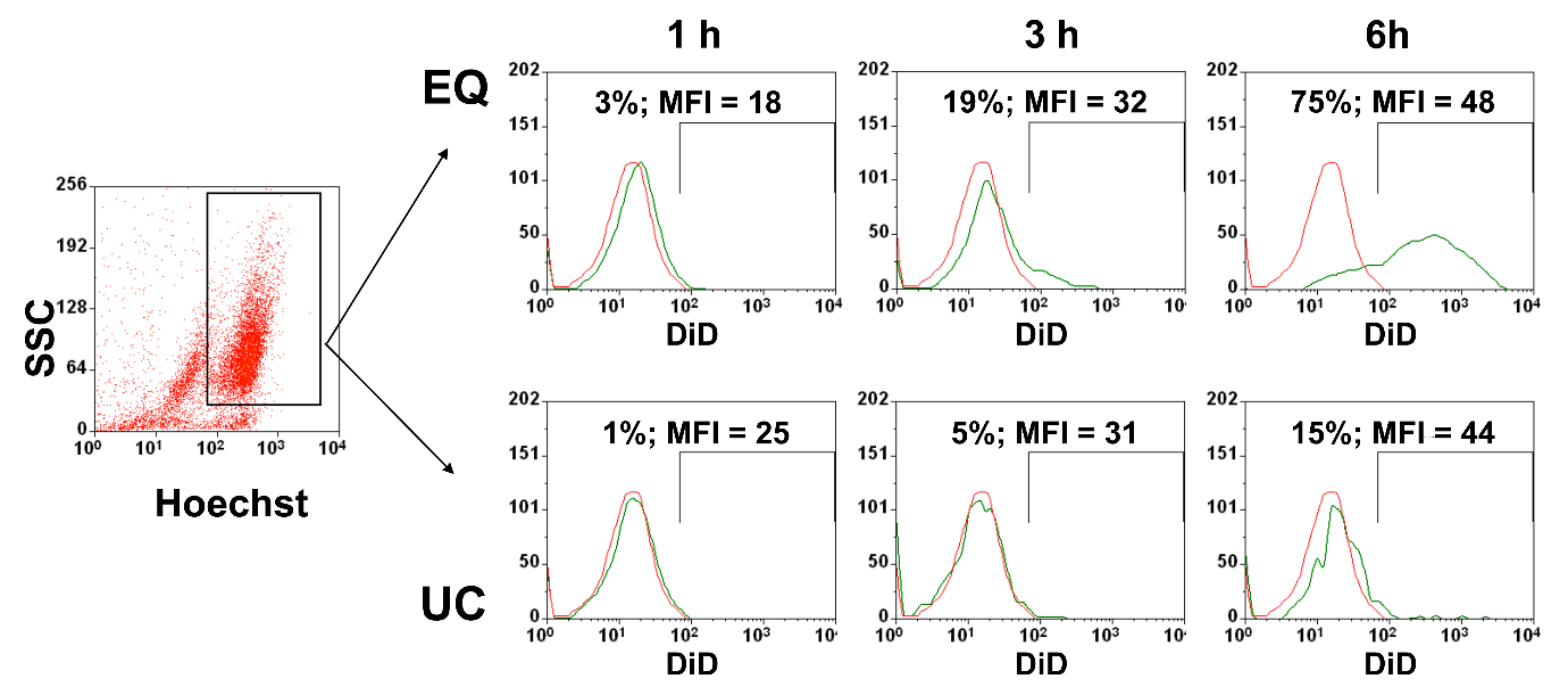


Figure 4

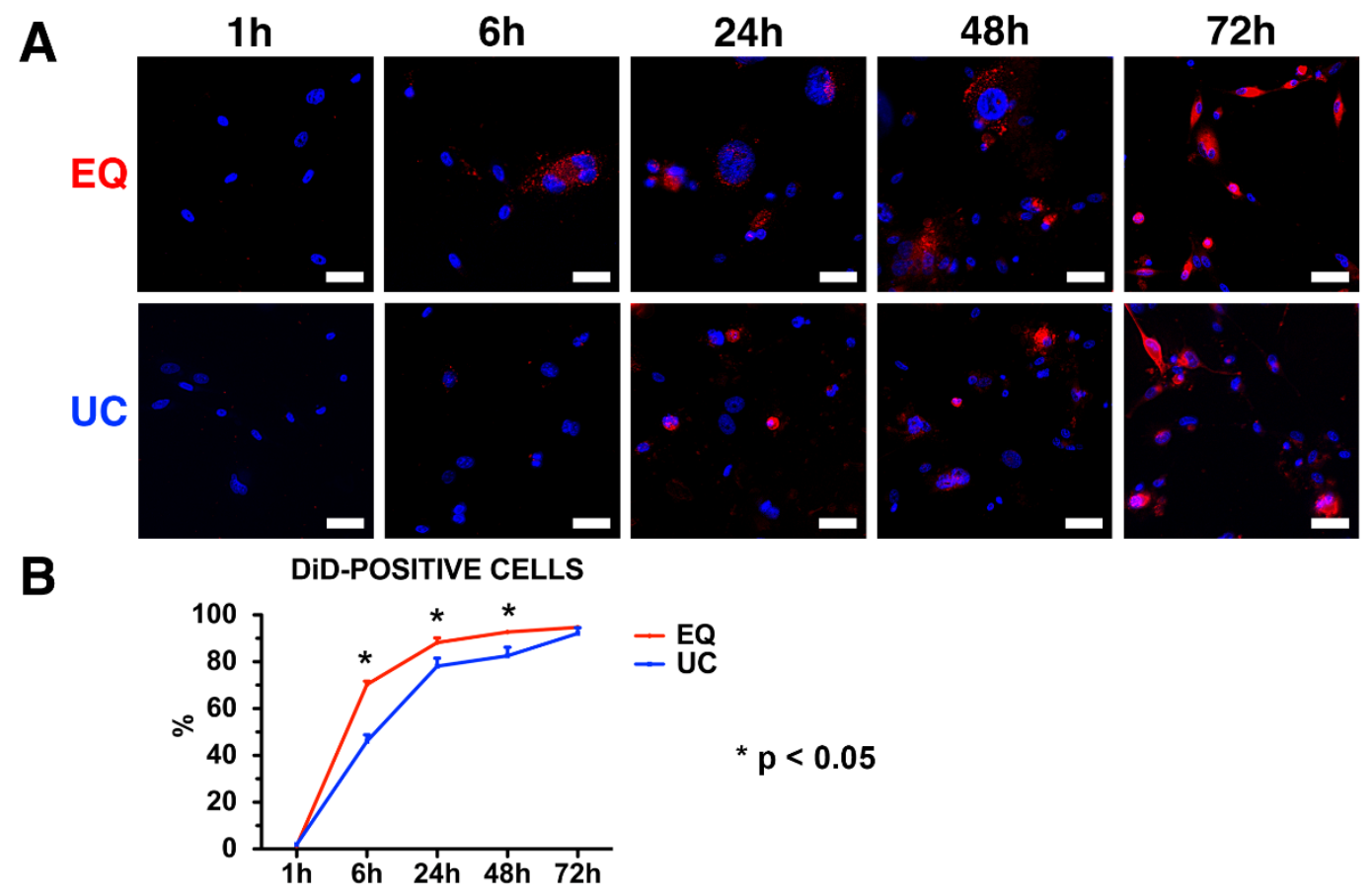

Figure 5

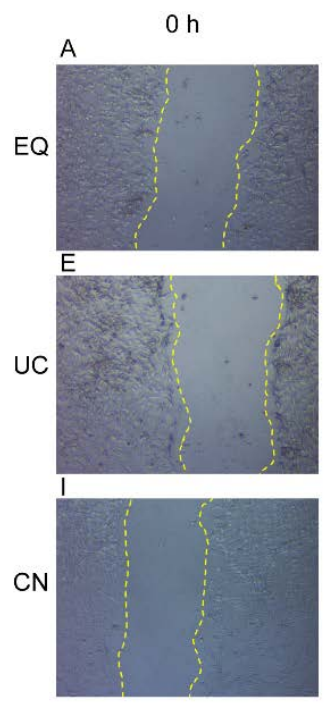

M
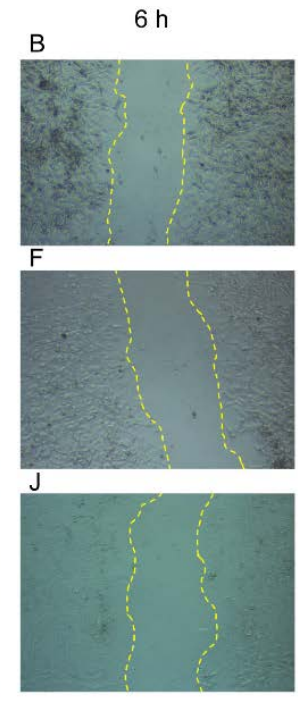

N

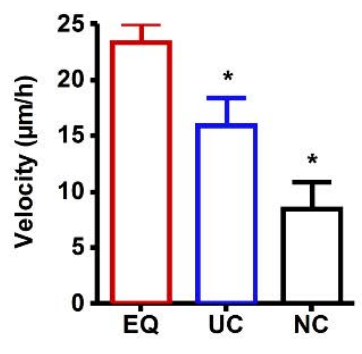

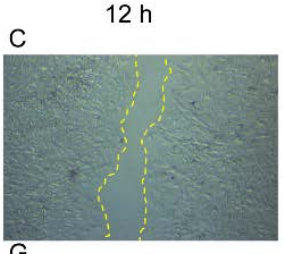
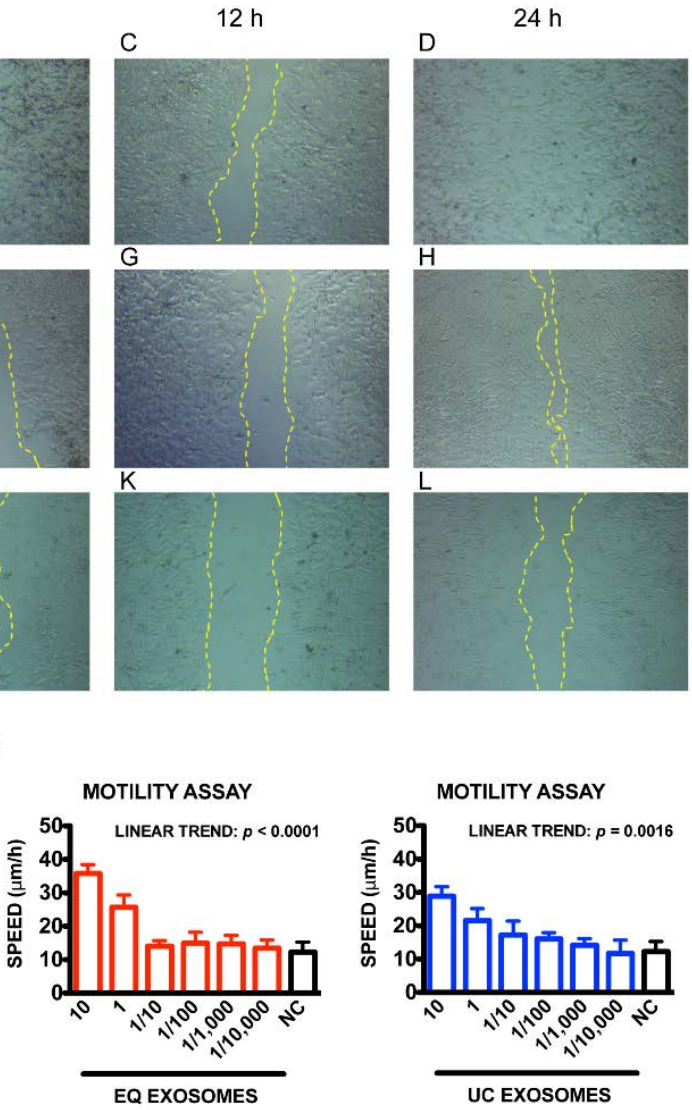